\title{
Investigation of Interaction between Vortices and Cavitation in a Turbulent Shear Layer*
}

\author{
Takashi OHTA**, Hiroki SAKAI***, Kie OKABAYASHI ${ }^{* * *}$ \\ and Takeo KAJISHIMA ${ }^{* * *}$ \\ ** Department of Mechanical Engineering, University of Fukui \\ 3-9-1 Bunkyo, Fukui 910-8507, Japan \\ E-mail: t-ohta@u-fukui.ac.jp \\ ${ }^{* * *}$ Department of Mechanical Engineering, Osaka University \\ 2-1 Yamadaoka, Suita, Osaka 565-0871, Japan
}

\begin{abstract}
We performed numerical simulations of a non-steady separated shear layer with and without cavitation to examine the effect on flow dynamics. Particular focus was given to the interaction between vortices and cavitation. In the simulations, streamwise and spanwise vortices generated by the instability of a shear layer flow, typical features in such a flow field, were formed further upstream in cavitating than in non-cavitating flows. In cavitating flows, the vortex shedding frequency and the Reynolds stresses increase, and we observed vortex weakening caused by cavitation. We found that a Burgers-type vortex can become the origin of a cavity. Contrarily, the growth of the cavity attenuates the corresponding vortex. We described this influence of a cavity on a streamwise vortex with a simple inviscid model.
\end{abstract}

Key words : Cavitation, Vortex, Shear Layer Flow, Turbulent Flow, Numerical Simulation

\section{Introduction}

Cavitation is known to have a significant effect on turbulent flow. Both large-scale and small-scale cavities can have major influence on the flow field and on vortices within it. Although much work has been done on sheet, cloud, blade-tip and other large-scale cavitation, models and analytical methods still need to be developed for cavitation on the small scale. To predict the characteristics of turbulence with cavitation, we need to know the interaction between vortices and cavities and the overall impact on the flow field. Large-scale roll vortices occur intermittently in a shear layer because of the Kelvin-Helmholtz instability. Streamwise vortices then arise in the shear layer extending between roll vortices ${ }^{(1),(2)}$. Katz and O'Hern ${ }^{(3)}$ reported that streamwise vortices can contribute to the generation of cavitation. It was confirmed $^{(3)-(5)}$ that cavities are generated when the pressure dips below the vapor pressure in the center of vortices.

Understanding cavitation is important practically because it can lead to improved performance of fluid machinery. Chen and Heister ${ }^{(6)}$ proposed a two-phase cavitation model with a parameter that needed to be found empirically, and Okita and Kajishima ${ }^{(7)}$ advanced a modified form of that model. Simulation methods were proposed ${ }^{(7)-(13)}$ to study the performance of fluid machinery in non-steady cavitating flows. Simulations that include the cavitation model of Okita and Kajishima have been reasonably successful in predicting experimental trends. Their model was used in simulations of 2D and 3D unsteady cavitating flows in cascades ${ }^{(7),(8),(12)}$ as well as in inducers ${ }^{(8),(12)}$. When compared with experimental results $^{(14)}$, the 3D simulation for unsteady cavitating flow in a cascade predicted reasonably well the dependence of drag and lift forces on cavitation number ${ }^{(7)}$. The simulation for a 3D flow in an inducer predicted vortex rotation frequencies that were consistent with experimental

*Received 6 Sep., 2011 (No. 11-0548) [DOI: 10.1299/jfst.6.1021]

Copyright (c) 2011 by JSME 
results $^{(12)}$.

Numerical analyses of shear layer flows have been studied using large-eddy simulations (LES) with cavitation models assuming turbulent flow ${ }^{(15),(16)}$. To our knowledge, however, previous simulation methods for cavitating flows have focused on large-scale low-pressure regions, such as a wake behind an object, and do not include the effects of interaction between cavities and small-scale turbulence structures. New subgrid scale (SGS) models for LES that include these effects are needed for more reliable predictions of turbulent flow. Recently, as a basic study for such models, Okabayashi and Kajishima ${ }^{(17)}$ proposed an estimation method of pressure fluctuation related to SGS vortices for prediction of cavitation inception in a turbulent mixing layer.

In this study, we performed numerical simulations of flow in a separated shear layer with non-steady three-dimensional vortices using the cavitation model of Okita and Kajishima ${ }^{(7)}$. We borrowed some ideas from the experimental setup of Iyer and Ceccio ${ }^{(18)}$ but used different conditions. Our purpose is to study and model the effect of cavities on streamwise vortices in our simulation from the viewpoint of vortex dynamics, and to contribute to the development of SGS and non-steady cavitation models that can reproduce a wide variety of flows from laminar to turbulent in the future.

\section{Theory}

We start with the mass conservation for a liquid,

$$
\frac{\partial\left(f_{\mathrm{L}} \rho_{\mathrm{L}}\right)}{\partial t}+\frac{\partial\left(f_{\mathrm{L}} \rho_{\mathrm{L}} u_{i}\right)}{\partial x_{i}}=0,
$$

where $f_{\mathrm{L}}$ is the volumetric fraction of the liquid phase, $\rho_{\mathrm{L}}$ is the density, and $u_{i}(i=1,2,3)$ are the components of the fluid velocity. We treat $\rho$ and $u_{i}$ as dimensionless variables normalized to a density scale $\rho_{0}$ and velocity scale $U_{0}$, such that the dimensional density $\rho^{*}$ is given by $\rho_{\mathrm{L}}^{*}=\rho_{\mathrm{L}} \rho_{0}$ and the dimensional velocity by $u^{*}=u U_{0}$. The situation $f_{\mathrm{L}}<1$ indicates a mixture of liquid and vapor.

We model the liquid density as having two components: an average density of 1 and a fluctuation $\rho_{\mathrm{L}}^{\prime}$, such that $\rho_{\mathrm{L}}=1+\rho_{\mathrm{L}}^{\prime}$. Equation (1) then leads to

$$
\frac{D f_{\mathrm{L}}}{D t}+f_{\mathrm{L}}\left(\frac{1}{1+\rho_{\mathrm{L}}^{\prime}} \frac{D \rho_{\mathrm{L}}^{\prime}}{D t}+\frac{\partial u_{i}}{\partial x_{i}}\right)=0
$$

For isentropic flow, the fluid density and static pressure $p^{*}$ are related via

$$
\frac{d p^{*}}{d \rho_{\mathrm{L}}^{*}}=a^{* 2},
$$

where $a^{*}$ is the speed of sound. Using a normalized pressure $p$, defined by $p^{*}=p \rho_{0} U_{0}^{2}$, and assuming the Mach number $M=U_{0} / a^{*}$ to be uniform, the variation of $\rho$ and $p$ takes the form

$$
\frac{d \rho_{\mathrm{L}}^{\prime}}{d t}=M^{2} \frac{d p}{d t}
$$

If we now assume a density fluctuation that is much less than the average density $\left(\rho_{\mathrm{L}}^{\prime} \ll 1\right)$, the mass conservation equation becomes

$$
\frac{D f_{\mathrm{L}}}{D t}+f_{\mathrm{L}}\left(M^{2} \frac{D p}{D t}+\frac{\partial u_{i}}{\partial x_{i}}\right)=0
$$

In deriving the equation of motion, we begin with the momentum conservation condition,

$$
\frac{\partial u_{i}}{\partial t}+u_{j} \frac{\partial u_{i}}{\partial x_{j}}=-\frac{1}{f_{\mathrm{L}}} \frac{\partial p}{\partial x_{i}}+\frac{\partial \tau_{i j}}{\partial x_{j}}
$$

where the viscous stress components $\tau_{i j}$ for a Newtonian fluid are given by

$$
\tau_{i j}=\frac{1}{R e}\left(\frac{\partial u_{i}}{\partial x_{j}}+\frac{\partial u_{j}}{\partial x_{i}}-\frac{2}{3} \delta_{i j} \frac{\partial u_{k}}{\partial x_{k}}\right),
$$


where $R e$ is the Reynolds number. If the divergence of the fluid velocity is small enough to be negligible, Eq. (6) then takes the form

$$
\frac{\partial u_{i}}{\partial t}+u_{j} \frac{\partial u_{i}}{\partial x_{j}}=-\frac{1}{f_{\mathrm{L}}} \frac{\partial p}{\partial x_{i}}+\frac{1}{R e} \frac{\partial^{2} u_{i}}{\partial x_{j}^{2}} .
$$

If the liquid is assumed to be incompressible, numerical instabilities arise because expansion and contraction of cavities in the surrounding fluid cause pressure changes ${ }^{(6)}$. Therefore, we allowed for compressibility by applying the low-Mach-number approximation ${ }^{(19)}$ to Eqs. (5) and $(8)$ in the simulation.

Chen and Heister ${ }^{(6)}$ modeled the expansion and contraction of cavities with the equation

$$
\frac{D \rho}{D t}=C\left(p-p_{\mathrm{v}}\right)
$$

where $C$ was to be determined empirically. The vapor pressure $p_{\mathrm{v}}$ is determined by the cavitation number,

$$
\sigma=\frac{p_{\infty}-p_{\mathrm{v}}}{\frac{1}{2} \rho_{\mathrm{L}} u_{\infty}^{2}}
$$

where $p_{\infty}$ and $\rho_{\mathrm{L}}$ are the pressure and liquid phase density, respectively, in the far field where there is no cavitation. The model of Chen and Heister allows the fluid density to decrease in the region where the pressure falls below the vapor pressure. Okita and Kajishima ${ }^{(7)}$ proposed a modified version of this equation,

$$
\frac{D f_{\mathrm{L}}}{D t}=\left\{C_{\mathrm{g}}\left(1-f_{\mathrm{L}}\right)+C_{\mathrm{l}} f_{\mathrm{L}}\right\}\left(p-p_{\mathrm{v}}\right)
$$

where $C_{\mathrm{g}}$ and $C_{1}$ are also empirically determined. We use Eq. (11) to model the evolution of cavities because of the previous predictive success ${ }^{(7),(12)}$ of simulations that include it.

\section{Simulation Procedure}

\subsection{Numerical Method}

Our computational method is the fractional step method used by Kajishima et al. ${ }^{(20)}$ and Okita and Kajishima ${ }^{(7)}$ with a collocated grid for incompressible flow. We calculated the velocity at a fractional step, $u_{i}^{\mathrm{F}}$, from the velocity at time step $n, u_{i}^{(n)}$, by approximating the spatial differential term in the equation of motion as a second-order difference. The velocity after a time interval $\Delta t$ was found for the next step using the pressure gradient:

$$
u_{i}^{(n+1)}=u_{i}^{\mathrm{F}}-\Delta t \frac{1}{f_{\mathrm{L}}} \frac{\partial p}{\partial x_{i}} .
$$

Substituting Eq. (12) into Eq. (5) gives the pressure equation:

$$
\frac{D f_{\mathrm{L}}}{D t}+f_{\mathrm{L}}\left\{M^{2}\left(\frac{\partial p}{\partial t}+u_{j} \frac{\partial p}{\partial x_{j}}\right)+\frac{\partial u_{j}^{\mathrm{F}}}{\partial x_{j}}-\Delta t \frac{\partial}{\partial x_{j}}\left(\frac{1}{f_{\mathrm{L}}} \frac{\partial p}{\partial x_{j}}\right)\right\}=0
$$

We solved this equation using a relaxation method by discretizing the spatial differential with the second-order central difference and applying the three-step method to the temporal difference.

$$
\text { Discretizing Eq. (12) leads to }
$$

$$
u_{i}^{(n+1)}=u_{i}^{\mathrm{F}}-\Delta t \frac{1}{f_{\mathrm{L}}^{(n)}} \delta_{i} p^{(n+1)}
$$

for the velocity. By introducing the three-step method for time integration and the secondorder central finite difference method for the spatial difference, one obtains

$$
\begin{aligned}
& \frac{D f_{\mathrm{L}}}{D t}+f_{\mathrm{L}}^{(n)}\left\{M ^ { 2 } \left(\frac{3 p^{(n+1)}-4 p^{(n)}+p^{(n-1)}}{2 \Delta t}\right.\right.\left.+u_{j}^{(n)} \delta_{j} p^{(n+1)}\right) \\
&\left.+\delta_{j} u_{j}^{\mathrm{F}}-\Delta t \delta_{j}\left(\frac{1}{f_{\mathrm{L}}^{(n)}} \delta_{j} p^{(n+1)}\right)\right\}=0
\end{aligned}
$$




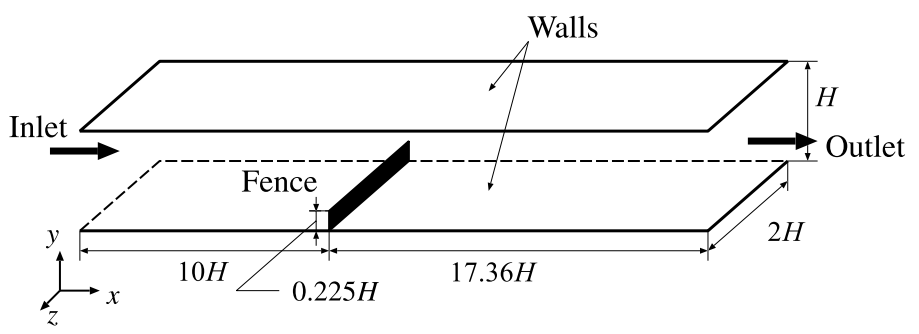

Fig. 1 Overview of the computational domain.

for the pressure equation (see Ref. (7) for more details).

As was done by Okita and Kajishima ${ }^{(7)}$, the liquid phase volumetric fraction for the $n$th time step was set to

$$
f_{\mathrm{L}}^{\mathrm{P}}=f_{\mathrm{L}}^{(n)}+\Delta t\left\{C_{\mathrm{g}}\left(1-f_{\mathrm{L}}\right)+C_{\mathrm{l}} f_{\mathrm{L}}\right\}^{(n)}\left(p^{(n)}-p_{\mathrm{v}}\right) .
$$

For $f_{\mathrm{L}}^{\mathrm{P}}<1$, the condition for cavitation, the volumetric fraction at the fractional step, $f_{\mathrm{L}}^{\mathrm{F}}$, was set to

$$
f_{\mathrm{L}}^{\mathrm{F}}=f_{\mathrm{L}}^{(n)}+\Delta t\left\{C_{\mathrm{g}}\left(1-f_{\mathrm{L}}\right)+C_{\mathrm{l}} f_{\mathrm{L}}\right\}^{(n)}\left(p^{(n+1)}-p_{\mathrm{v}}\right),
$$

and, for $f_{\mathrm{L}}^{\mathrm{P}} \geq 1$,

$$
f_{\mathrm{L}}^{\mathrm{F}}=1 \text {. }
$$

The volumetric fraction at the next time step was then found from the effect of convection:

$$
f_{\mathrm{L}}^{(n+1)}=f_{\mathrm{L}}^{\mathrm{F}}-\Delta t u_{j}^{(n+1)} \frac{\partial f_{\mathrm{L}}}{\partial x_{j}} .
$$

In the simulations, we used a clipping ( $f_{\mathrm{L}} \geq 0.1$ ) so that Eqs. (5) and (8) would not fail.

This way, we obtained the pressure, $p^{(n+1)}$, velocity, $u_{i}^{(n+1)}$, and liquid phase volumetric fraction, $f_{\mathrm{L}}^{(n+1)}$, after time marching with a time-step interval, $\Delta t$. Non-steady flow was then calculated by repeating the above procedure.

\subsection{Parameters and Boundary Conditions}

In the experiment of Iyer and $\mathrm{Ceccio}^{(18)}$, a plate with a sharp edge causes a shear layer perpendicular to the flow. However, we used a simpler shape for the flow field in our simulation. Figure 1 shows a schematic of the computational domain. Using the channel width $H$ as the unit of length, the streamwise length of the parallel channel was $L_{x} / H=27.36$, and the spanwise length was $L_{z} / H=2$. A fence of height 0.225 units and zero thickness was placed 10 units from the inflow boundary to generate a shear layer.

Chen and Heister ${ }^{(6)}$ found that results did not vary much over a very wide range of values for $C$. What matters in choosing values for $C_{\mathrm{g}}$ and $C_{\mathrm{l}}$ is that they satisfy the type of cavity development desired. Numerical optimization gave the values $C_{\mathrm{g}}=1000$ and $C_{\mathrm{l}}=1$ when $p<p_{\mathrm{v}}$, which implies that cavities are forming and growing. For contraction and vanishing, the values were $C_{\mathrm{g}}=100$ and $C_{\mathrm{l}}=1$ with $p>p_{\mathrm{v}}$. We used a uniform Mach number of 0.1 (as did Okita and Kajishima ${ }^{(7)}$ ) and a time step of $5 \times 10^{-5}$. We simulated a single-phase flow field and the flow field for the cavitation number $\sigma=0.5$.

For the inflow boundary condition, the velocity profile was made quadratic with respect to the wall normal and was uniform in the spanwise direction. The bulk velocity was $U_{0}$. At the outflow boundary, we applied the convective condition

$$
\frac{\partial u_{i}}{\partial t}+u_{\mathrm{c}} \frac{\partial u_{i}}{\partial x_{1}}=\frac{1}{R e} \frac{\partial^{2} u_{i}}{\partial x_{j}^{2}},
$$

where the convection velocity, $u_{\mathrm{c}}$, is the average of the maximum and the minimum of the velocity at the outflow boundary. We used a non-reflection condition proposed by Okita and 


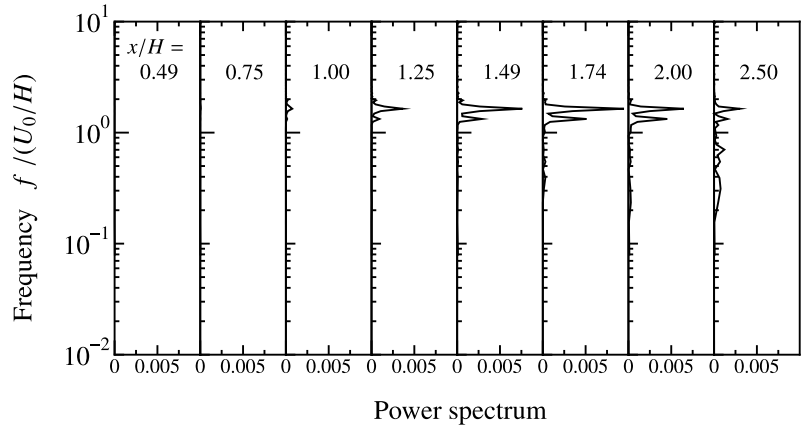

(a) Single phase flow

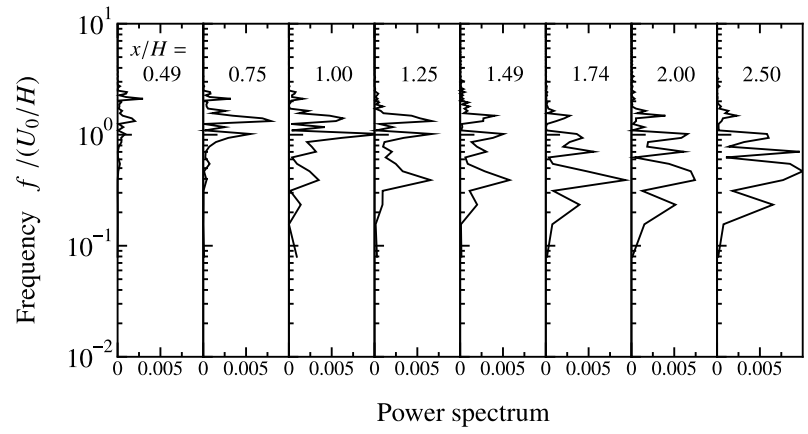

(b) Flow with cavitation $(\sigma=0.50)$

Fig. 2 Influence of cavitation on the frequency of spanwise vortex shedding.

Kajishima $^{(7)}$ for pressure at the inflow and outflow boundaries, and used no-slip conditions for velocity at the walls. A periodic condition was used in the spanwise direction.

The number of grid points for discretization was 640 in the streamwise direction (defined as the $x$ direction), where 256 grid points were located upstream from the fence and 384 downstream. These points increased in concentration as one approached the fence. Ninety-six grid points were located uniformly in the wall-normal direction ( $y$ direction) and 160 in the spanwise direction ( $z$ direction). The $x, y$ and $z$ directions are orthogonal to each other. The minimum grid spacing in the streamwise direction was $\Delta x / H=1.61 \times 10^{-2}$ and the maximum was $\Delta x / H=5.77 \times 10^{-2}$. The grid spacings in the wall-normal and spanwise directions were $\Delta y / H=1.04 \times 10^{-2}$ and $\Delta z / H=1.25 \times 10^{-2}$, respectively. The Reynolds number was $R e=H U_{0} / v=1 \times 10^{4}$.

\section{Results}

In the following, $\overline{()}$ and ()$^{\prime}$ indicate the mean value and the fluctuation of each quantity, respectively. The computational results are normalized with respect to the length, $H$, the velocity, $U_{0}$, and the liquid density, $\rho_{\mathrm{L}}$, in the flow field of the single phase.

\subsection{Modulation of a Shear Layer by Cavitation}

A roll vortex is formed in the shear layers of the simulation. Spectral analysis of velocity fluctuations can identify the cycle in which the vortex is formed, the results of which are shown in Fig. 2. In this analysis, the velocity components normal to the streamwise direction were averaged in the spanwise direction at one point in the shear layer. Then, the spectral analysis was executed for the temporal variation of the averaged velocity components. The release frequency of a roll vortex, $f$, can be estimated using the peak of the spectrum. For the singlephase flow, $f /\left(U_{0} / H\right)=1.4$ and is independent of the position in the range of our simulations. However, for cavitating flow $(\sigma=0.50)$, the frequency is $f /\left(U_{0} / H\right)=2.0$ at $x / H=0.49$ downstream of the fence, which is larger than that of single-phase flow. Furthermore, the 


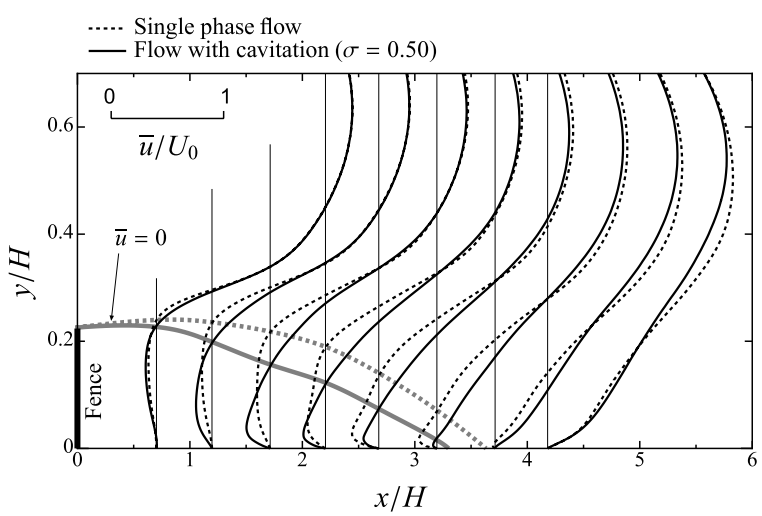

Fig. 3 Profiles of the streamwise mean velocity, $\bar{u} / U_{0}$. From this, the development of shear layer thickness can be characterized.

spectrum frequency band tends to broaden in the cavitating flow.

In Fig. 2, the frequency of velocity fluctuations increases while moving upstream. This might be because the trigger of the instability in the shear layer appears upstream. We attribute this tendency in our study to the disturbance induced by the strong instability of the entire cavitation field. In Fig. 3, we can see that the thickness of the shear layer develops early, because the locations of roll vortices move upstream. Consequently, the reattachment point of the separation from the fence also moves upstream.

Figures 4-6 show the contour plots for the liquid fraction, Reynolds stresses and vorticity, respectively, of the flow field for the single-phase and cavitating flows. Figure 5 shows the contours of the Reynolds stresses, $\overline{u^{\prime} u^{\prime}}, \overline{v^{\prime} v^{\prime}}$ and $\overline{w^{\prime} w^{\prime}}$, and Reynolds shear stress, $-\overline{u^{\prime} v^{\prime}}$, where $u, v$ and $w$ are the streamwise, vertical and spanwise velocity, respectively. Figure 6 shows the contours for $\overline{\omega_{x}^{\prime 2}}$ and $\overline{\omega_{z}^{\prime 2}}$, where $\omega_{x}$ and $\omega_{z}$ are the streamwise and spanwise component of velocity, respectively. All of these contours are downstream from the fence. In our simulations, the turbulent intensities for the cavitating flow are distributed upstream for all contours of stress and vorticity when compared with those for single-phase flow, even though the change of the intensities has no constant tendency. In Fig. 6(a), the secondary streamwise vortices between roll vortices cannot be observed statistically because of the intermittency.

\subsection{Correlation between a Streamwise Vortex and Cavitation in a Shear Layer}

Figure 7 depicts a single-phase instantaneous flow field, while Fig. 8 depicts an instantaneous flow field in the presence of cavitation. To visualize the vortices in the three-dimensional flow field, we used isosurfaces of the second invariant of the velocity gradient tensor,

$$
Q=\frac{1}{2}\left(W_{i j} W_{i j}-S_{i j} S_{i j}\right)
$$

Here, $W_{i j}$ and $S_{i j}$ are the asymmetric and symmetric components of the velocity gradient tensor, respectively. The location of the fence is marked by the black regions in the upstream cross-section shown in the figures. From this, we confirmed that roll vortices induced by the instability of a shear layer and the secondary generated streamwise vortices were reproduced in the simulations.

Cavities are distributed such that they overlap with the vortices, as shown in Fig. 8(b). Cavities corresponding to the streamwise vortices can be observed in the figure. Cavities corresponding to the roll vortices are also present, but not all of them are visually represented by the isosurfaces at the threshold used here. Under cavitation conditions, many roll vortices are distributed upstream, and the secondary streamwise vortices are also concentrated upstream in comparison to the single-phase case. This tendency is in good agreement with the features of the power spectrum and the turbulent statistics mentioned above. 


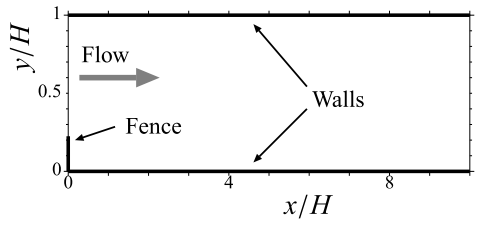

single phase

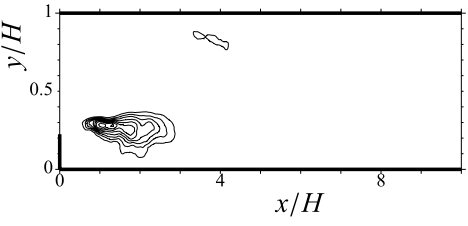

cavitation $(\sigma=0.5)$

Fig. 4 Contours of the liquid fraction, $\bar{f}_{\mathrm{L}}$. Contour increment is 0.01 .

(a) $\overline{u^{\prime} u^{\prime}} / U_{0}^{2}$

$\underset{\Xi}{\beth}$
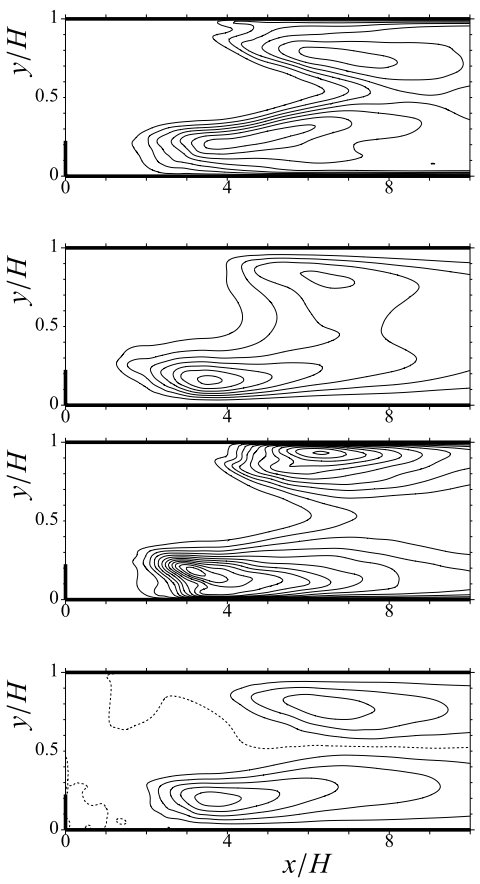

single phase
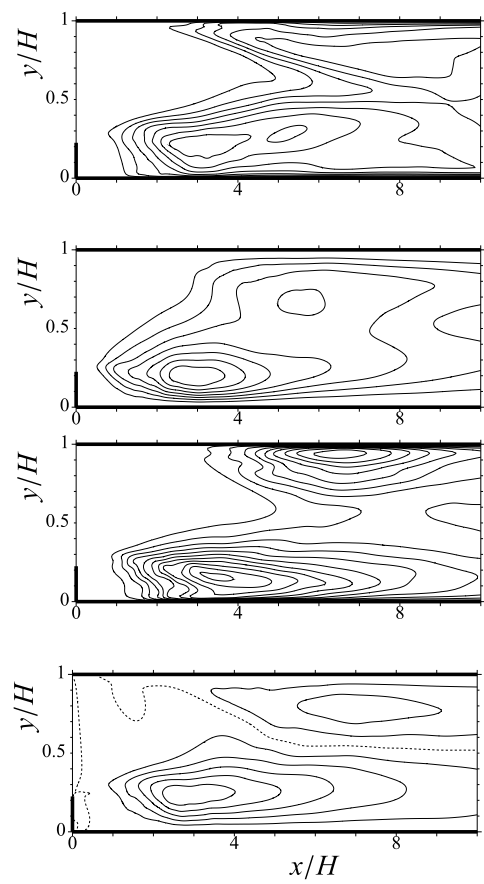

cavitation $(\sigma=0.5)$

Fig. 5 Contours of the Reynolds stresses, (a) $\overline{u^{\prime} u^{\prime}} / U_{0}^{2}$, (b) $\overline{v^{\prime} v^{\prime}} / U_{0}^{2}$ and (c) $\overline{w^{\prime} w^{\prime}} / U_{0}^{2}$, and Reynolds shear stress, (d) $-\overline{u^{\prime} v^{\prime}} / U_{0}^{2}$. Contour increment is (a) 0.01 , (b) 0.002 , (c) 0.002, (d) 0.004 .
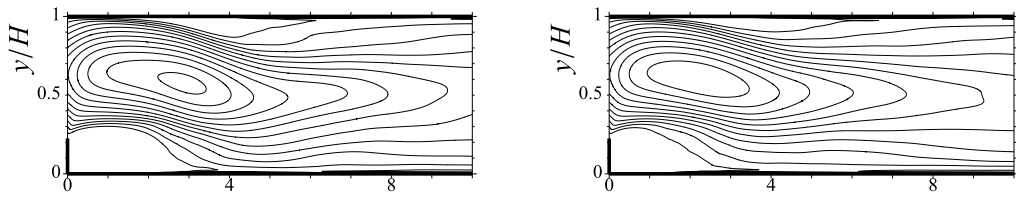

(a) $\overline{\omega_{x}^{\prime 2}} /\left(U_{0} / H\right)^{2}$
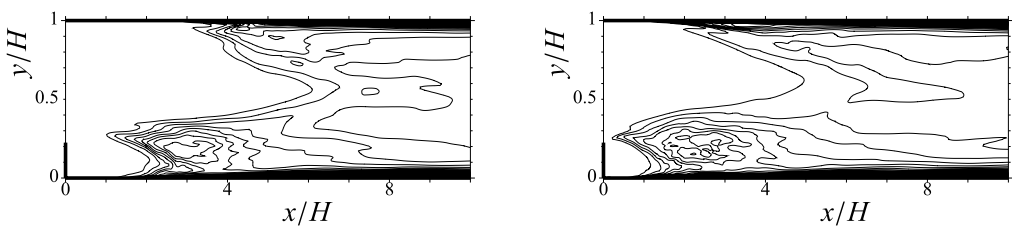

single phase

Fig. 6 Contours of vorticity fluctuation intensities (a) $\overline{\omega^{\prime 2}} /\left(U_{0} / H\right)^{2}$ and (b) $\overline{\omega_{z}^{\prime 2}} /\left(U_{0} / H\right)^{2}$. Contour increment is (a) 10 , (b) 2 . 


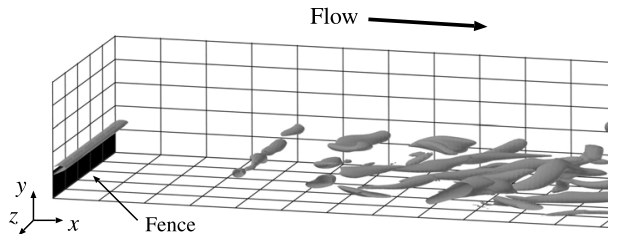

Fig. 7 Overview of an instantaneous flow field of single phase (isosurfaces of $\left.Q /\left(U_{0} / H\right)^{2}=10\right)$

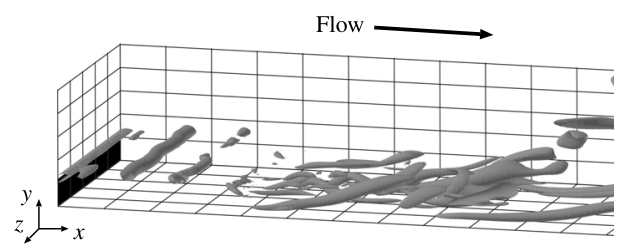

(a) Isosurfaces of $Q /\left(U_{0} / H\right)^{2}=10$

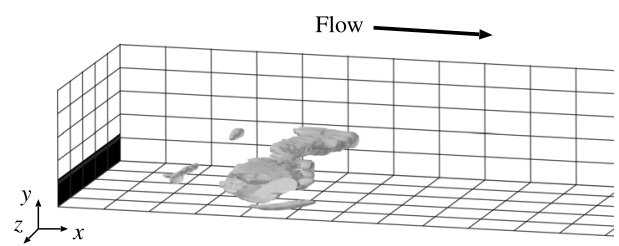

(b) Isosurfaces of $f_{\mathrm{L}}=0.99$

Fig. 8 Overview of an instantaneous flow field with cavitation $(\sigma=0.50)$.
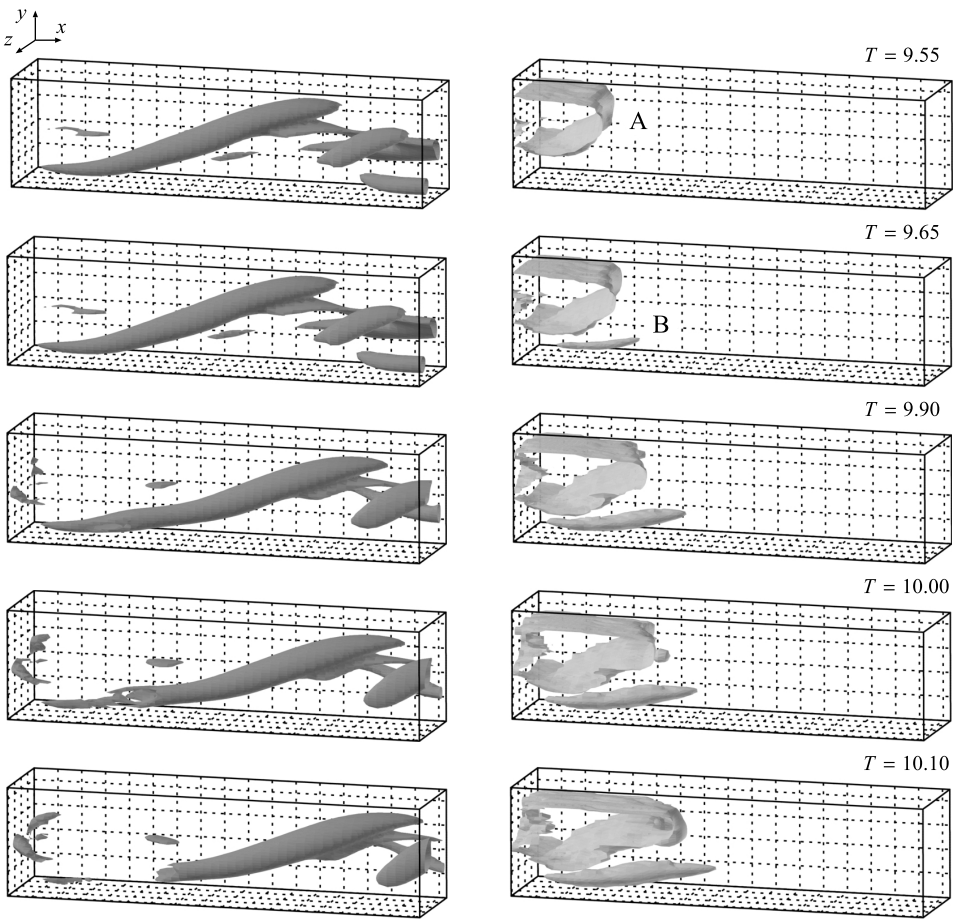

(a) Vortices $\left(Q /\left(U_{0} / H\right)^{2}=10\right)$

(b) Cavities $\left(f_{\mathrm{L}}=0.99\right)$

Fig. 9 Closeup view of a streamwise vortex and a cavity. 
The relation of vortices to cavitation is of special interest. Figure 9 shows the temporal variation in appearances of a streamwise vortex and a cavity. Here, $T=t /\left(H / U_{0}\right)$ is defined as the dimensionless time. Vortices and cavities are visualized using isosurfaces of the second invariant of the velocity gradient tensor, $Q /\left(U_{0} / H\right)^{2}=10$, and the volumetric fraction of the liquid phase, $f_{\mathrm{L}}=0.99$, respectively. Although a streamwise vortex is observed in this region at $T=9.55$, there is no region of $f_{\mathrm{L}} \leq 0.99$ in the vortex, only a cavity corresponding to the upstream roll vortex. This vortex is marked at point A in Fig. 9.

However, the roll vortex cannot be visualized with the threshold used for $Q$. The pressure upstream of the streamwise vortex was below the vapor pressure. At $T=9.65$, a cavity appeared in the center region of the streamwise vortex, which is marked point B (see Fig. 9). This investigation suggests that the low pressure region in the streamwise vortex serves as the origin of a cavity. Furthermore, the cavity size increases as time passes. In Fig. 9(a), we see from the isosurface that the shape of the streamwise vortex becomes irregular upstream. After $T=9.90$, the cavity continues to grow, but the streamwise vortex vanishes before $T=10.10$. These changes in vortex behavior seem to be unique to vortex cavitation, because they are not observed in single-phase flow. In the case of single-phase flow, there are only viscous diffusion and dissipation effects that weaken vortices.

In summary, a cavity is formed after the pressure in the center region of a streamwise vortex falls below the vapor pressure; the generated cavity then attenuates the vortex.

\subsection{Temporal Variation of a Streamwise Vortex with Cavitation}

Next, we investigated a streamwise vortex that was weakened by the growth of a cavity. Figures 10 and 11 show the temporal variations of the relative circumferential velocity profile, $u_{\theta}-u_{\theta 0}$, and the pressure distribution in a streamwise vortex versus the distance from the center of the vortex, $z-z_{0}$, respectively, over the interval $T=9.55-10.10$. The profiles are along the line in the spanwise direction through the center of the vortices. The velocity component aligns to the surface normal of the central axis of the vortex. When tracking a vortex, which is moving and deforming temporally, the center of the vortex can be determined based on the maximum point of the second invariant of the velocity gradient tensor, $Q$. The position of the cross-section was chosen so that the distance from the upstream edge of the isosurface to $Q /\left(U_{0} / H\right)^{2}=10$ was constant.

The circumferential velocity profiles, at $T=9.55$ just before cavitation and at $T=9.65$ just after cavitation are similar to that of the Burgers vortex. Therefore, the minimum of pressure is at the center of the vortex. The gradient of the circumferential velocity at the center, which corresponds to the difference between the maximum and the minimum velocities, diminishes at $T=9.90$ when the cavity is generated and begins to grow. Simultaneously, the pressure at the center rises and approaches the vapor pressure. At $T=10.00$ and $T=10.10$, the gradient of the velocity becomes smaller after the cavity has grown. This is because both the pressure gradient and density of the gas-liquid mixture are small in the cavitation region. Then the velocity profile, which determines the shape of the centrifugal force, balances the small pressure gradient. The pressure in the center is still below the vapor pressure. In Fig. 12, which shows the profiles of the streamwise component of vorticity, $\omega_{x}$, we see that the vorticity gradually diminishes with the growth of the cavity.

\section{Discussion}

\subsection{Comparisons to Previous Studies}

We have observed some common phenomena between our results and those of previous studies even with different conditions. Qualitatively, the spectrum frequency in our simulation is similar to that in experiments conducted by Young and Holl ${ }^{(21)}$ and Belahadji et al. ${ }^{(22)}$, in which cavitation in the body wake increased the frequency of wake vortices. However, the experimental conditions in those studies differed from those of our simulations. Our simulations also show much greater weakening of the streamwise vortex for the cavitating flow than 


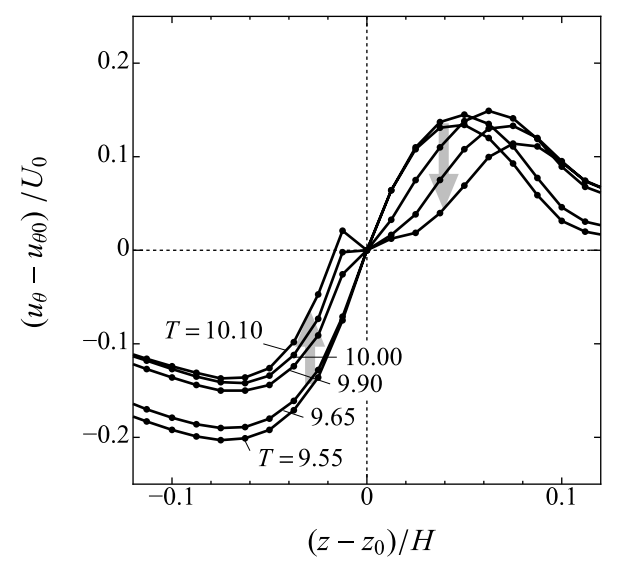

Fig. 10 Variation of circumferential velocity profile of a streamwise vortex. $\left(u_{\theta 0}\right.$ is velocity at the core, and $z_{0}$ is the position of the core)

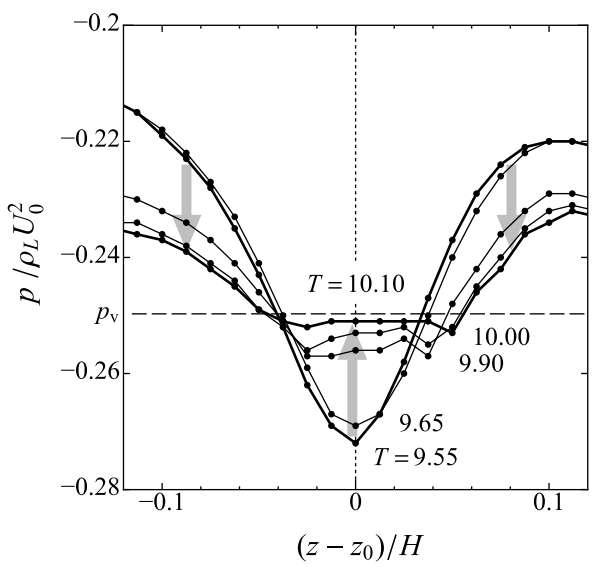

Fig. 11 Variation of pressure profile in a streamwise vortex. (the horizontal dashed line is vapor pressure, $p_{\mathrm{v}}$ )

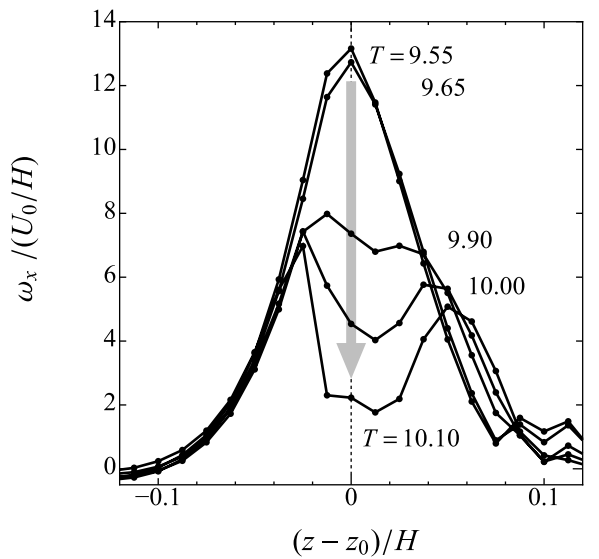

Fig. 12 Variation of the vorticity profile in a streamwise vortex. 


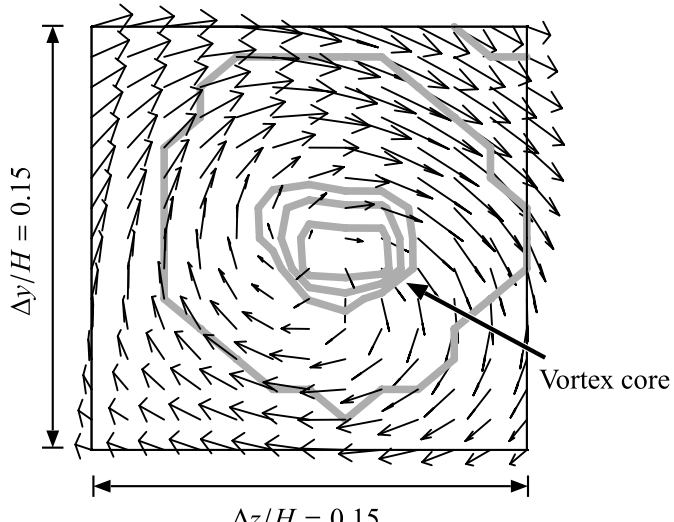

Fig. 13 Closeup view of instantaneous velocity vectors and contours of $f_{\mathrm{L}}$ for the $(y, z)$ cross-section of a cavitating streamwise vortex at $T=9.90$.

in the single-phase flow, which coincides with the growth of a cavity. When Xing et al. ${ }^{(23)}$ simulated a flow with a vortex ring from a round jet using conditions different from ours, the cavitation generated in the vortex ring transformed and destroyed the vortex ring. These results indicate a similar tendency of the variation of a streamwise vortex. In the experiment of Iyer and Ceccio ${ }^{(18)}$, the growth and flow behavior of the shear layer were affected by the growth and collapse of cavities, which our simulation also found. Iyer and Ceccio experimentally confirmed that the cavities affect the turbulent intensity in the region downstream from the cavitating flow field ${ }^{(18)}$.

As an example of previous studies related to the change of the circumferential velocity of a vortex by cavitation, there was an observation of cavitation in a blade tip vortex in weak water by Arndt and Keller ${ }^{(24)}$. They showed, through an analysis of a blade tip vortex modeled as a two-dimensional Rankin vortex, that the maximum circumferential velocity increased $40 \%$ when a gas phase was present in the center of the vortex with constant circulation. However, in our simulations, the circumferential velocity decreases. This discrepancy could be caused by the fact that the Rankin vortex assumed by Arndt and Keller does not include the effect of the radial transportation of the liquid phase. In other words, in the absence of the liquid phase displacement outside the inspection volume, the circumferential velocity increases because of the conservation of circulation. In these simulations, however, flow toward the outside of the vortex core was observed, and can be seen in Fig. 13. If the liquid phase is pushed outward along the radial direction and circulation is kept constant, circumferential velocity should decrease.

\subsection{Model for Predicting a Vortex with Cavitation}

To predict the changes in a streamwise vortex caused by cavitation in the simulation, we constructed a model that expresses the variation of the circumferential velocity during cavitation. Here, we assume the phenomenon to be two-dimensional on a plane normal to the central axis of a vortex. In Fig. 14, the liquid particles are on a circle of radius $r$ in relation to the vortex core just before cavitation, and their circumferential velocity is $u_{\theta}(r)$. After a cavity grows radially from the vortex core, the liquid particles at radius $r$ are pushed out to radius $r+\Delta r$, and their circumferential velocity becomes $u_{\theta}^{\prime}(r+\Delta r)$. For the period in which the cavity is expanding, we can assume that liquid regions are pushed out only in the radial direction. The circulation, $\Gamma(r)$, at radius $r$ before cavitation is

$$
\Gamma(r)=2 \pi r u_{\theta}(r) .
$$

Similarly, the circulation after cavitation, $\Gamma^{\prime}(r+\Delta r)$, is

$$
\Gamma^{\prime}(r+\Delta r)=2 \pi(r+\Delta r) u_{\theta}^{\prime}(r+\Delta r)
$$




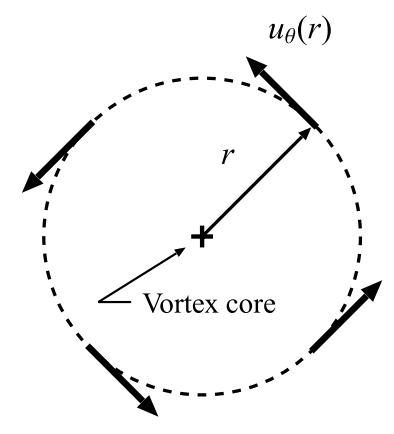

(a) Before cavity expansion

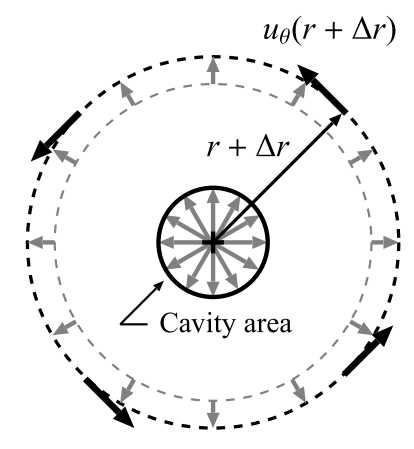

(b) After cavity expansion

Fig. 14 Schematics of a modeled expanding cavity.

If the influence of the viscosity is negligible and the circulation is conservative while the cavity is expanding, then:

$$
u_{\theta}^{\prime}(r+\Delta r)=\frac{r}{r+\Delta r} u_{\theta}(r) .
$$

Assuming that the cavity expands near the vortex core and the liquid phase within radius $R_{\mathrm{c}}$ is pushed outward,

$$
\pi(r+\Delta r)^{2}-\pi r^{2}=\pi R_{\mathrm{c}}^{2},
$$

then the radial length of the displacement $\Delta r$ is

$$
\Delta r=\sqrt{r^{2}+R_{\mathrm{c}}^{2}}-r .
$$

The variation of the circumferential velocity caused by cavitation can be derived from Eqs. (24) and (26). Then, we assume that the gas-phase region within an inspection region, $D$, that includes a vortex is concentrated in the region of the vortex core. Also, the area that the cavity pushes outward, $A_{\mathrm{c}}=\pi R_{\mathrm{c}}^{2}$, can be estimated using an area integral of the volumetric fraction of the gas-phase region, $f_{\mathrm{G}}=1-f_{\mathrm{L}}$, within $D$ :

$$
A_{\mathrm{c}}=\int_{D}\left(1-f_{\mathrm{L}}\right) d S \text {. }
$$

We compared a vortex predicted with our model to a cavitating vortex that was formed in the separated shear layer in the simulation. To estimate $A_{\mathrm{c}}$, we used the volumetric fraction of the liquid phase, $f_{\mathrm{L}}$, at each time. We took the value of $u_{\theta}$ from the velocity profile of the simulation at $T=9.55$, before cavitation, and used it to predict the velocity profile during cavity expansion using our model. Next, we compared the predicted velocity profile during cavity expansion to the results of the simulation.

Figure 15 shows the profiles of the circumferential velocity predicted using this model. The velocity tends to decrease as the cavity grows and the gradient of the profile decreases near the center. The profiles predicted for the streamwise vorticity component using the inviscid model are shown in Fig. 16. As the cavity grows, the vorticity decreases in the center and the profile forms a plateau. These predictions are in good agreement with the results of the simulation shown in Figs. 10 and 12. Although the method used to estimate the radius $R_{\mathrm{c}}$ is arbitrary, our simple model for the circumferential velocity accurately expresses the vortical structure. This structure corresponds to the profiles of $u_{\theta}$ and $\omega_{x}$, which are important for predicting the turbulent energy and the energy dissipation rate.

\section{Conclusions}

We performed non-steady simulations of a turbulent shear layer with cavitation to observe the interaction between vortices and cavitation in turbulent flow. The cavitation model we used has had prior success in predicting experimental behavior. In our simulations, the 


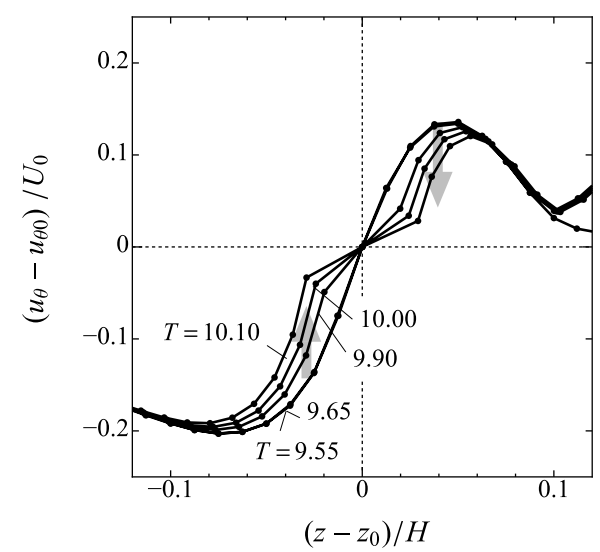

Fig. 15 Variation of predicted circumferential velocity profile.

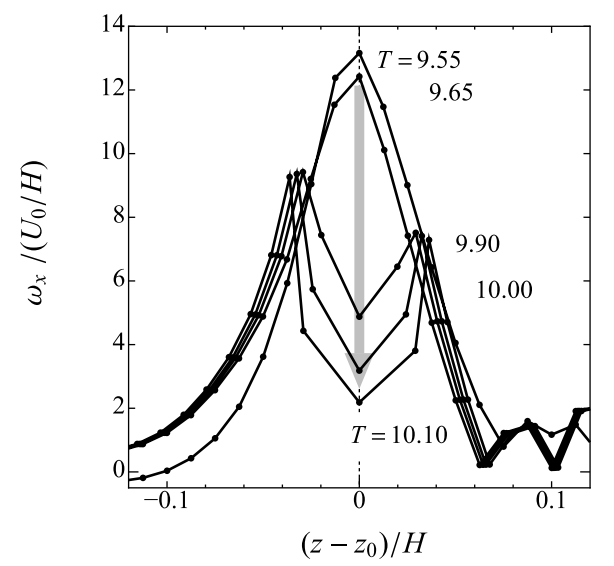

Fig. 16 Variation of predicted streamwise vorticity profile.

cavities expanded and contracted according to the difference between the pressure and the vapor pressure. The target of the simulations was the shear layer that was separated from a fence that was installed in the parallel channel. Roll vortices and streamwise vortices induced by the instability of a shear layer were reproduced in the simulations. We then confirmed that not only a roll vortex but also a streamwise vortex could be the origin of a cavity, by capturing cavitation generated in its low-pressure regions. We also found the following, while accounting for the interaction between cavitation and the flow field:

- The position where roll vortices are formed moves upstream, because of the instability in the shear layer with cavitation, and the frequency of vortex shedding just downstream of the fence increased in comparison to the single-phase case. Thus, this aids the development of the shear layer.

- Through the generation of cavitation, the instability of the entire flow field becomes strong and the Reynolds stress increases.

- The growth of cavities in streamwise vortices affects the profile of the circumferential velocity and weakens the streamwise vortices.

- The simple inviscid model proposed in this study can model the effects on a vortex caused by the growth of a cavity.

Because cavitation can change the microscopic characteristics of turbulent flow and the macroscopic properties of the flow field, large-eddy simulation of non-steady cavitating flow without fine resolution scales might be insufficient. A new subgrid scale model that accounts for the influence of vortices on cavities and the behavior of vortices that are weakened by 
cavitation is needed.

\section{References}

( 1 ) Breidenthal, R., Response of Plane Shear Layers and Wakes to Strong Threedimensional Disturbances, Physics of Fluids, Vol.23, No.10 (1980), pp.1929-1934.

( 2 ) Lasheras, J. C. and Choi, H., Three-dimensional Instability of a Plane Free Shear Layer: An Experimental Study of the Formation and Evolution of Streamwise Vortices, Journal of Fluid Mechanics, Vol.189 (1988), pp.53-86.

( 3 ) Katz, J. and O'Hern, T. J., Cavitation in Large Scale Shear Flows, Journal of Fluids Engineering, Vol.108, No.3 (1986), pp.373-376.

( 4 ) Katz, J., Cavitation Phenomena within Regions of Flow Separation, Journal of Fluid Mechanics, Vol.140 (1984), pp.397-436.

( 5 ) O'Hern, T. J., An Experimental Investigation of Turbulent Shear Flow Cavitation, Journal of Fluid Mechanics, Vol.215 (1990), pp.365-391.

( 6 ) Chen, Y. and Heister, S. D, Two-phase Modeling of Cavitated Flows, Computers $\mathcal{E}$ Fluids, Vol.24, No.7 (1995), pp.799-809.

( 7 ) Okita, K. and Kajishima, T., Three-dimensional Computation of Unsteady Cavitating Flow in a Cascade, Proceedings of the 9th International Symposium on Transport Phenomena and Dynamics of Rotating Machinery, (2002), No.FD-ABS-076 (CD-ROM).

( 8 ) Okita, K., Matsumoto, Y. and Kamijo, K., Numerical Analysis for Unsteady Cavitating Flow in a Pump Inducer, Proceedings of the 5th International Symposium on Cavitation, Osaka, Japan, (2003).

( 9 ) Iga, Y., Nohmi, M., Goto, A. and Ikohagi, T., Numerical Analysis of Cavitation Instabilities Arising in the Three-Blade Cascade, Journal of Fluids Engineering, Vol.126, No.3 (2004), pp.419-429.

(10) Ohashi, Y. and Kajishima, T., Numerical Simulation of Rotating Cavitation in a 2-D Cascade, Proceedings of the 6th KSME-JSME Thermal E Fluids Engineering Conference, (2005), No.DG-01 (CD-ROM).

(11) Kimura, T., Yoshida, Y., Hashimoto, T. and Shimagaki, M., Numerical Simulation for Unsteady Cavitating Flow in a Turbopump Inducer, Proceedings of the 6th International Symposium on Cavitation, (2006), No.41 (CD-ROM).

(12) Ugajin, H., Kawai, M., Okita, K., Ohta, T. and Nakano, M., Numerical Analysis of the Unsteady Cavitating Flow in a 2D-cascade and a 3D-inducer, Proceedings of the 43rd AIAA/ASME/SAE/ASEE Joint Propulsion Conference, Cincinnati, USA, (2007).

(13) Fukaya, M., Tamura, Y. and Matsumoto, M., Prediction of Cavitation Intensity and Erosion Area in Centrifugal Pump by Using Cavitating Flow Simulation with Bubble Flow Model, Journal of Fluid Science and Technology, Vol.5, No.2 (2010), pp.305-316.

(14) Numachi, F., Tsunoda, H. and Chida, I., Cavitation Tests on Six Profiles for Blade Elements, Transactions of the Japan Society of Mechanical Engineers, Vol.17, No.60 (1951), pp.1-5, (in Japanese).

(15) Yamade, Y., Kato, C., Tani, N. and Yamanishi, N., Large Eddy Simulation of Cavitating Flows around a Two-dimensional Body, Proceedings of the 18th CFD Symposium, (2004), Vol.A7, No.2.

(16) Shams, E. and Apte, S. V., Prediction of Small-Scale Cavitation in a High Speed Flow Over an Open Cavity Using Large-Eddy Simulation, Journal of Fluids Engineering, Vol.132, No.11 (2010), 111301.

(17) Okabayashi, K and Kajishima, T., Modeling of the Subgrid-Scale Pressure Distribution in Turbulent Mixing Layer, Journal of Fluid Science and Technology, Vol.6, No.1 (2011), pp.73-84.

(18) Iyer, C. O. and Ceccio, S. L., The Influence of Developed Cavitation on the Flow of a Turbulent Shear Layer, Physics of Fluids, Vol.14, No.10 (2002), pp.3414-3431.

(19) Inagaki, M., Murata, O., Kondoh, T. and Abe, K., Numerical Prediction of Fluid- 
Resonant Oscillation at Low Mach Number, AIAA Journal, Vol.40, No.9 (2002), pp.1823-1829.

(20) Kajishima, T., Ohta, T., Okazaki, K. and Miyake, Y., High-Order Finite-Difference Method for Incompressible Flows Using Collocated Grid System, JSME International Journal, Series B, Vol.41, No.4 (1998), pp.830-839.

(21) Young, J. O. and Holl, J. W., Effects of Cavitation on Periodic Wakes behind Symmetric Wedges, Journal of Basic Engineering, Vol.88, (1966), pp.163-176.

(22) Belahadji, B., Franc, J. P. and Michel, J. M., Cavitation in the Rotational Structures of a Turbulent Wake, Journal of Fluid Mechanics, Vol.287 (1995), pp.383-403.

(23) Xing, T., Li, Z. and Frankel, S. H., Numerical Simulation of Vortex Cavitation in a Three Dimensional Submerged Transitional Jet, Journal of Fluids Engineering, Vol.127, No.4 (2005), pp.714-725.

(24) Arndt, R. E. A. and Keller, A. P., Water Quality Effects on Cavitation Inception in a Trailing Vortex, Journal of Fluids Engineering, Vol.114, No.3 (1992), pp.430-438. 